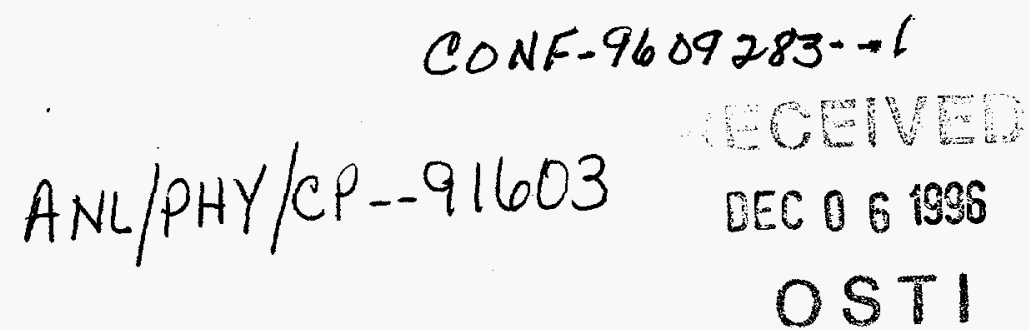

The submitted manuscript has been created by the University of Chicago as Operator of Argonne National Laboratory ("Argonne") under Contract No. W-31-109-ENG-38 with the U.S. Department of Energy. The U.S. Government retains for itself, and others acting on its behalf, a paid-up, nonexclusive. irrevocable worldwide license in said article to reproduce, prepare derivative works, distribute copies to the public, and perform publichy and display publicly, by or on behalf of the Government.

\title{
Extending Synchrotron-Based Atomic Physics Experiments into the Hard X-Ray Region
}

\author{
T. LeBrun
}

Physics Division, Argonne National Laboratory, Argonne, Illinois 60439, USA

\begin{abstract}
The high-brightness, hard $\mathrm{x}$-ray beams available from third-generation synchrotron sources are opening new opportunities to study the deepest inner shells of atoms, an area where little work has been done and phenomena not observed in less tightly bound inner-shells are manifested. In addition scattering processes which are weak at lower energies become important, providing another tool to investigate atomic structure as well as an opportunity to study photon/atom interactions beyond photoabsorption. In this contribution we discuss some of the issues related to extending synchrotron-based atomic physics experiments into the hard $\mathrm{x}$-ray region from the physical and the experimental point of view. We close with a discussion of a technique, resonant Raman scattering, that may prove invaluable in determining the spectra of the very highly-excited states resulting from the excitation of deep inner shells.
\end{abstract}

\section{Introduction}

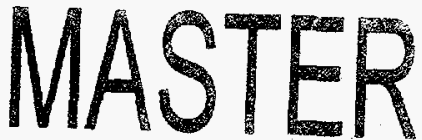

Synchrotron sources are evolving rapidly and driving changes in x-ray optics and experimental techniques that are transforming the capabilities of synchrotronbased experiments. The very bright and energetic $x$-ray beams available at thirdgeneration sources allow the wide array of measurements performed using VUV photoexcitation to be extended into the x-ray region where new phenomena are expected. In addition, the range of high photon energies includes a domain where scattering processes compete strongly with and even dominate photoabsorption, shifting focus for atomic physics experiments to include photon/atom scattering in the hard $\mathrm{x}$-ray region. The photon beams from third generation sources such as the APS, the ESRF and Spring-8 are well suited to such studies because the high photon energies open the deepest inner-shells of the periodic table to investigation and the high brightness compensates for the small interaction cross sections while allowing more detailed investigations via highly differential measurements and coincidence techniques.

The structure and relaxation dynamics of the deepest inner shells has received much less attention than those shells accessible in the VUV, and this contribution discusses some of the physical and instrumental implications of the shift from low energy to high energy synchrotron-based atomic physics experiments. The author would like to take this opportunity to recognize Paul Cowan's contributions as a scientist, collaborator and a friend. All of the results discussed here were taken at 


\section{DISCLAIMER}

This report was prepared as an account of work sponsored by an agency of the United States Government. Neither the United States Government nor any agency thereof, nor any of their employees, makes any warranty, express or implied, or assumes any legal liability or responsibility for the accuracy, completeness, or usefulness of any information, apparatus, product, or process disclosed, or represents that its use would not infringe privately owned rights. Reference herein to any specific commercial product, process, or service by trade name, trademark, manufacturer, or otherwise does not necessarily constitute or imply its endorsement, recommendation, or favoring by the United States Government or any agency thereof. The views and opinions of authors expressed herein do not necessarily state or reflect those of the United States Government or any agency thereof. 


\section{DISCLADMER}

Portions of this document may be illegible in electronic image products. Images are produced from the best available original document. 
beamline X24A at the NSLS, a facility whose great productivity testifies to Paul's ongoing contributions to many areas of synchrotron-based research.

\section{Background}

Valence shell atomic spectra have been actively studied for more than a century and have made essential contributions to such advances as the development of the quantum theory, quantum electrodynamics, and the understanding of atomic structure. While studies of inner-shell atomic spectra also began early, it was the introduction of tunable synchrotron-based light sources in the VUV and x-ray region of the electromagnetic spectrum that sparked development of the field.

The wide range and tunability of the available photon energies prompted a new generation of experiments probing the behavior of atoms and molecules at excitation energies above, and well beyond the ionization threshold. These synchrotron-based experiments were unique because the tunability and intensity of synchrotron radiation allowed quasi-discrete states near ionization thresholds to be selectively excited and their decay studied. These studies revolutionized our understanding of the structure and dynamics of atomic inner shells, illuminating such phenomena as the photoelectric effect, the interaction of discrete and continuum states (autoionization), giant resonances in scattering continua, and correlations in strongly interacting many body systems.

But only half of the subshells of neutral atoms have excitation energies less than one keV. Higher photon energies probe more tightly bound shells where the strong nuclear potential increases the importance of relativistic effects (1) and Kshell electrons may be sensitive to nuclear size effects or couple to nuclear magnetic moments - effects visible in X-ray spectra (2). When one also considers systems such as the rich variety of negative ions which have their transitions shifted to even higher energies, the importance of $x$-rays to atomic structure studies becomes evident. Thus far, however, the exploration of resonant excitation of atoms by hard $x$-rays remains in its infancy.

\section{$X$-ray Interactions}

We will begin by briefly discussing the characteristics and magnitudes of the $x$ ray/atom interaction processes which are dominant for photon energies up to 150 $\mathrm{keV}$, and then turn to experimental implications. The upper limit of $150 \mathrm{keV}$ has been selected here to include the absorption thresholds of the heaviest stable elements (e.g. U $1 \mathrm{~s}=115 \mathrm{keV}$ ), as well as higher energies where multiple corehole states far above thresholds are found and where Compton and Rayleigh scattering can dominate the attenuation cross section. Note that this range of energies is now fully accessible at currently operating synchrotron sources by using wigglers and wavelength shifters. Indeed, Compton scattering measurements have already been performed at $1 \mathrm{MeV}$ photon energy by Suortti and coworkers at the ESRF (3). For more information on these scattering processes the reader is referred to recent reviews by Crasemann (4) and Pratt $e t$. al. (5).

The interaction of a photon with an atom is traditionally distinguished by whether the photon is absorbed (photoabsorption), elastically scattered (also 
called Rayleigh or coherent scattering), or inelastically scattered (Compton or incoherent scattering). While these are convenient descriptions, they are not unambiguous because the absorption and re-emission of a photon is indistinguishable from scattering in experiments which are not time-resolved. As a result some processes which involve resonant photoexcitation can fall under more than one of these classifications, as we will show later. At the photon energies of interest here, scattering from electrons dominates atom/x-ray interactions so processes which are important at higher photon energies such as pair production and nuclear scattering will be neglected in our discussion for the sake of brevity. For discussions of nuclear scattering see the contributions by Smirnov, and Kikuta in these proceedings.

Concentrating on electronic scattering, we then have the following processes to consider:

- Photoabsorption (followed by Auger or fluorescent decay)

$$
\gamma+A_{0} \rightarrow\left\{\begin{array}{c}
A_{i}^{*} \\
A_{i^{\prime}}^{*}+e_{p h}^{-}
\end{array}\right\} \rightarrow\left\{\begin{array}{c}
A_{f}^{*}+e_{A}^{-} \\
A_{f^{\prime}}^{*}+\gamma
\end{array}\right.
$$

Here the first set of braces indicates the highly-excited intermediate state that follows photoexcitation: a quasi-discrete state for resonant excitation (top row), or a state with a continuum of energy eigenvalues for photoionization (bottom row). The second set of braces shows the two competing decay mechanisms for the intermediate state: Auger decay (top) or fluorescence decay (bottom).

Photoabsorption is the dominant attenuation process at low to moderate $\mathrm{x}$-ray energies: it exceeds elastic scattering by 3-4 orders of magnitude near the $K$ threshold of light atoms like carbon and is 1-2 orders of magnitude stronger than elastic scattering near the $\mathrm{K}$ thresholds of heavier elements like lead. It is also a clean and selective form of excitation because the energy and angular momentum transferred to the target are well known when the dipole approximation is satisfied, however the widely-used dipole approximation must be applied cautiously in the $x$-ray region. Recent experimental results $(6,7)$ have verified earlier predictions that show photoelectron angular distributions measured at high photon energy or near threshold to be sensitive to interference between dipole and higher-order multipole transition amplitudes. These studies are just beginning, and measurements of non-dipole effects are likely to be an active area of research at high energy synchrotron sources (8).

- Elastic $=$ Rayleigh scattering

$$
\gamma+A_{0} \rightarrow A_{0}+\gamma
$$

Elastic scattering from atoms is also called Rayleigh scattering 1 , or coherent scattering because the scattered radiation has the same wavelength as the incident

\footnotetext{
1 Note that in contrast to Rayleigh scattering, Thompson scattering is elastic scattering for the idealized case of a single free charged particle, such as an electron or nucleus, when the recoil energy of the particle is ignored. Inclusion of the recoil kinematics leads to a description of Compton scattering which reduces to the Thompson formula at low photon energy where the recoil energy is small.
} 
radiation as well as a definite phase shift. Interference effects such as diffraction are therefore observed in elastic scattering. Because diffraction is such an important technique for structure determination, cross sections and angular distributions for elastic scattering are important to applications in structural biology and materials science. The data for these applications must cover a wide range of chemical elements and photon energies, and tables of calculated values are widely used. Away from threshold, currently available theoretical results compare well with experiment and serve the needs of experiments using traditional fixed-energy sources. However the tunability of synchrotron radiation allows crystallographers to obtain more information using scattering near thresholds (e.g. information regarding the phase of the structure factor) - an area where measurements to test theory are still needed. Indeed even the most complete calculations frequently use the independent particle approximation (9), which is expected to be less reliable near thresholds. The need for testing theory near threshold is striking in light of the rapid evolution of structural biology at synchrotron sources, and provides a good opportunity for synchrotron-based atomic and molecular science to make an important contribution while demonstrating it's role as a source of fundamental data for a wide range of fields of research.

- Inelastic $=$ Compton scattering + Raman scattering

$$
\gamma+A_{0} \rightarrow A_{f}^{*}+\gamma^{\prime}+e^{-}
$$

Compton scattering is photon scattering from a free electron, and in the range of photon energies we are considering it is the basic interaction process between an electron and a (real) photon. In the center-of-mass frame Compton scattering is just elastic scattering, but the transformation to laboratory coordinates gives rise to Doppler shifts and results in the well known characteristics of Compton scattering (10), for example the Compton shift of the wavelength of the scattered photon which depends only on the scattering angle and is independent of the incident photon energy. ${ }^{2}$

In scattering from atoms the electrons are of course not free and the effects of binding modifies Compton scattering in a number of ways. The cross section at low energy is reduced and the spectrum of scattered energies is broadened and slightly shifted. While simple kinematic approaches to binding effects serve well in many cases, the recent availability of second-order $S$ matrix calculations (11) has raised the bar for synchrotron-based experiments seeking to study rigorously Compton scattering.

Atomic structure is also manifested in inelastic channels which can be described as Compton scattering. For example, photoionization followed by fluorescence decay can be considered an inelastic scattering process, indeed the two are indistinguishable in an experiment that can not resolve the core-hole lifetime (12). Because this process corresponds to inelastic scattering with a free electron in the final state, it can clearly be described as a form of Compton scattering, however it is also a form of $\mathrm{x}$-ray Raman scattering - the term which

2 The Compton shift at $90^{\circ}$ is equal to $0.0243 \AA$, which is the wavelength of a photon whose energy equals $m \mathrm{c}^{2}$. Note that the change in energy of the Compton-scattered photon is not independent of the incident photon energy because energy is inversely proportional to wavelength. 
appears to be preeminent among the synchrotron research community. For more information on Compton scattering, as well as the relation between Compton and Raman scattering see Heitler (13), or the articles by Åberg (14) or Bergstrom (11).

\section{Experimental Implications}

As we have seen, photoabsorption dominates photon-atom interactions at energies near absorption thresholds. This implies that for light elements photoabsorption is most important at low energies (up to $20 \mathrm{keV}$ for carbon) while it contributes strongly to attenuation in heavier elements over the whole range of photon energies considered here (1-150 keV). We henceforth concentrate on experiments that use the photoeffect and the subsequent decay processes to study inner-shell structure. To illustrate the experimental consequences of doing such experiments with harder $x$-rays we will compare cross sections and natural widths for different elements, referencing the values to the threshold energy instead of $Z$ because most studies at synchrotrons are concerned with near-threshold excitation. Figure 1 illustrates the variation of cross sections at threshold for photoabsorption, Auger decay, and fluorescent decay as a function of the energy of the $\mathrm{K}$ and $\mathrm{L}_{3}$ absorption edges of the elements.

The lower left figure shows the magnitude of the $\mathrm{K}$ shell photoabsorption cross section for the elements neon to thorium $(Z=10-90)$ at the $K$ edge (15). The cross section for $\mathrm{K}$-shell ionization at threshold decreases by two orders of magnitude in going from $\mathrm{Ne}$ to $\mathrm{Th}$, and this highlights the need for very intense $\mathrm{X}$ ray sources in probing the deepest shells of elements. If the photoabsorption cross

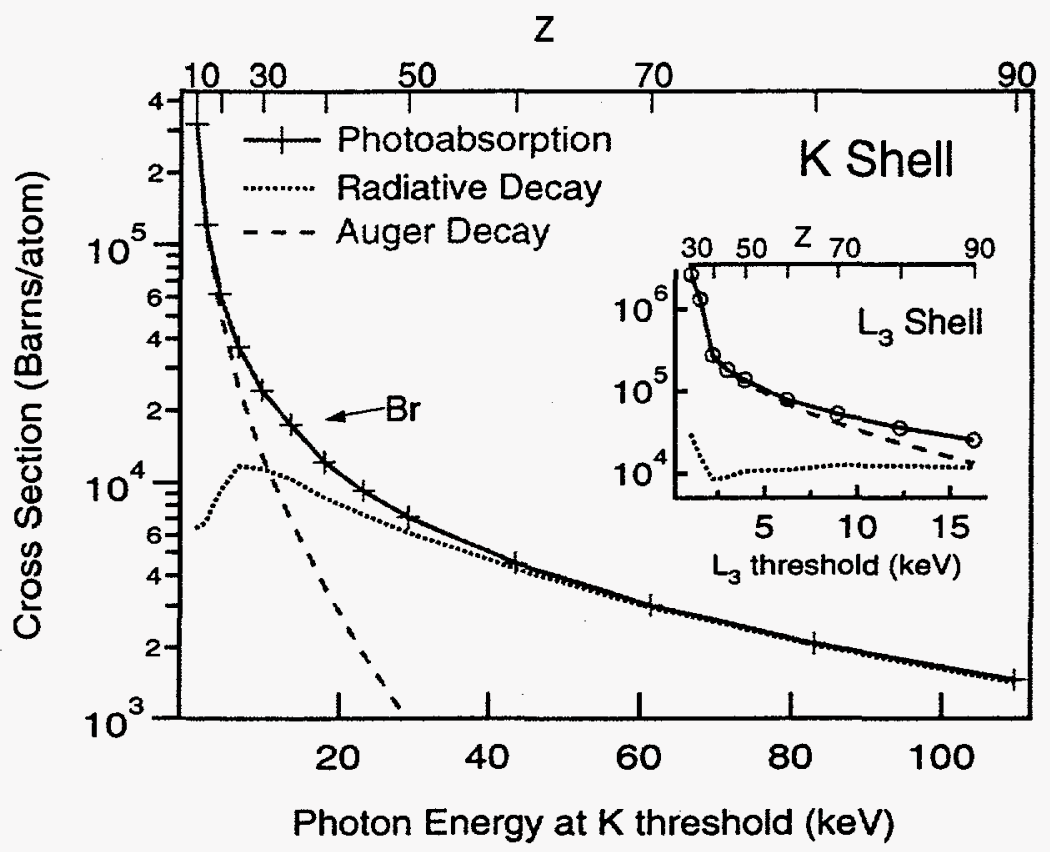

FIGURE 1. Cross sections at threshold for photoabsorption (solid line with crosses), Auger decay (dashes), and fluorescent decay (dots) for the $K$ shell and $L_{3}$ shell (inset) of the elements for $Z=10$ to 90 . 
sections are then multiplied by the radiative and nonradiative yields (16), one obtains the cross sections for fluorescence and Auger relaxation following $\mathrm{K}$-shell ionization. The plot shows that the intensity of Auger decay from a $\mathrm{K}$-hole falls much more rapidly than the photoabsorption cross section as $\mathrm{Z}$ is increased, while the intensity of $\mathrm{K}$ fluorescence varies less rapidly than the photoabsorption cross section. Therefore, though Auger decay is typically the dominant relaxation mechanism at low energy, radiative decay is stronger for $\mathrm{K}$ core-holes beyond 10 keV binding energy.

As a result, Auger spectroscopy of $\mathrm{K}$-hole relaxation in heavy elements poses a challenge because of the declining cross sections and the experimental difficulties associated with the required high fields and accompanying low transmission of the electron spectrometers. While the small spot sizes available from thirdgeneration synchrotron sources may help alleviate these problems, the strength of the radiative decay channels provides a complimentary approach which, combined with advances in high resolution $\mathrm{x}$-ray spectroscopy, increases the potential for application of $\mathrm{x}$-ray spectroscopy to atomic physics experiments at synchrotron facilities.

The fall-off of the Auger decay intensity is not as dramatic for other shells as shown in the inset where analogous data for the $L_{3}$ subshells is presented. Here Auger decay remains strong, but still decreases slightly faster than photoionization while fluorescence varies relatively little.

However, in determining the feasibility of experiments, intensity needs to be balanced with the required resolution. Some factors related to experimental resolution are summarized in figure 2 where the natural widths of core holes in different subshells of the elements sodium to uranium (17) are plotted versus the threshold energy of the subshell. The natural width of $\mathrm{K}$ holes varies from $0.3 \mathrm{eV}$

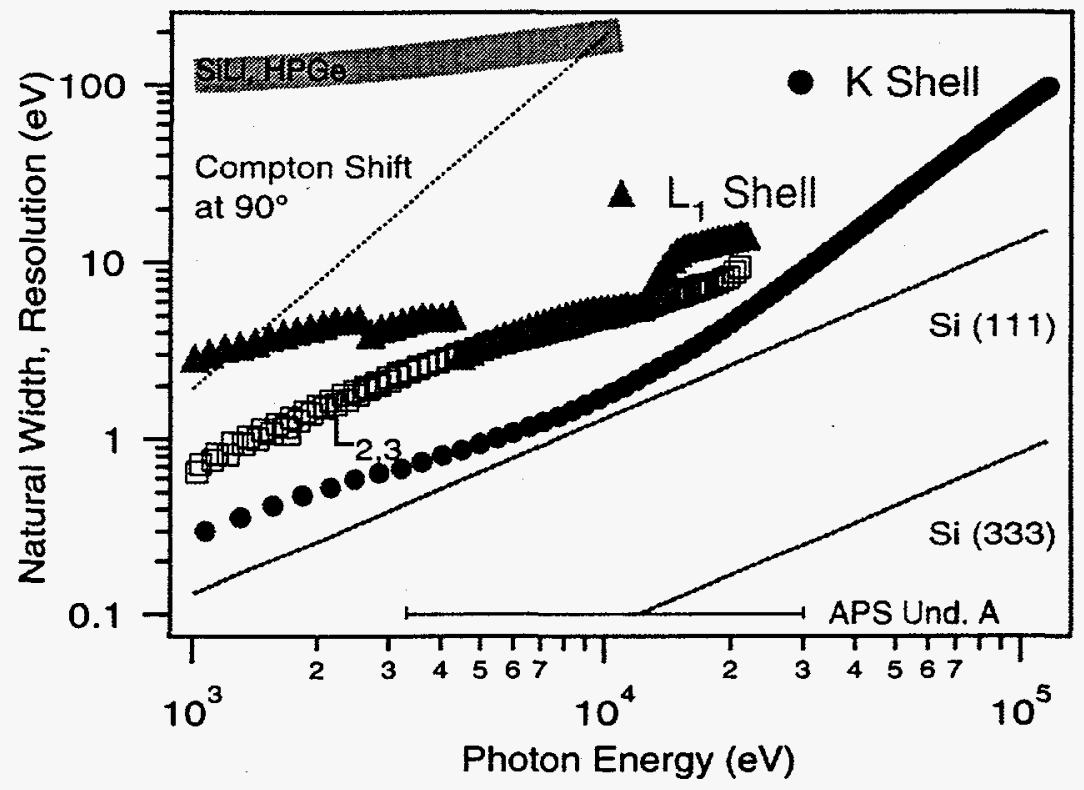

FIGURE 2. Natural widths of core holes for the elements sodium to uranium $(Z=11-92)$ plotted versus the ionization energy of the core hole. The theoretical resolution of selected crystal reflections and detectors are included for comparison. The other quantities in the plot are explained in the text. 
for $\mathrm{Na}$ to $96.1 \mathrm{eV}$ for $\mathrm{U}$, while the $\mathrm{L}_{1}$ natural width of $\mathrm{U}$ is roughly $14 \mathrm{eV}$. When these widths are compared to the resolution required to study resonance or Rydberg structure near threshold (less than $1 \mathrm{eV}$ ) it is clear that most of the nearedge features will be washed out by the natural width. As we shall discuss in the last section, resonant Raman spectroscopy may provide a route to studying nearedge structure with an effective resolution that is narrower than the lifetime width, so we include that possibility in this discussion of instrumental requirements.

For experiments such as photoabsorption and nonresonant photoelectron spectroscopy, a bandpass that matches the core-hole lifetime width or is slightly narrower is optimal for most experiments. The workhorse of many double crystal monochromators is the 111 reflection of silicon, and it is fortunate that the theoretical resolution of $\mathrm{Si}(111)$ is reasonably well matched to the lifetime widths (see Fig. 2), although flux-limited experiments in the L shells or high-Z K shells may benefit from more reflective crystals such as $\mathrm{Ge}$ (111).

In order to use resonant Raman scattering to resolve structure near threshold, spectral widths narrower than lifetime widths will be required. $\mathrm{Si}(111)$ will serve for $\mathrm{L}$ shell measurements at intermediate energies, but if the technique lives up to its promise, higher-order reflections (intrinsic resolution $\Delta \mathrm{E} / \mathrm{E}=10^{-5}$ or better) and high resolution post-monochromators will ultimately become necessary. These target values for the resolution also set tough standards for $\mathrm{x}$-ray and electron spectrometers, where the transmission falls rapidly for high resolution and high analyzing energy. Fortunately the $\mathrm{L}$ shell threshold energies for high $\mathrm{Z}$ elements fall within the range of energies available from the undulator $A$ at the Advanced Photon Source, indicating that insertion devices at third-generation sources can provide the higher intensities needed in the required range of photon energies.

Finally, in relation to scattering studies note that the Compton shift at $90^{\circ}$ is generally large compared to the natural width for energies beyond a few $\mathrm{keV}$, so high purity germanium or $\mathrm{Si}(\mathrm{Li})$ detectors can resolve Compton and Rayleigh scattering above approximately $10 \mathrm{keV}$. Such detectors can also be very useful in coincidence experiments which only need to distinguish relaxation channels between different subshells.

\section{Resonant Raman Scattering}

It is clear from the above considerations that lifetime broadening will limit efforts to study structure near absorption edges of deep inner-shells. However resonant Raman scattering has recently emerged as a promising technique to address this limitation. This stems from the fact that the natural width of the initial core hole does not explicitly contribute to the linewidths of fluorescence or Auger lines following resonant excitation, thus resonant peaks are narrower than peaks resulting from ionization. This is exploited in resonant inelastic $x$-ray scattering to study the short-lived intermediate states that result from core excitation. The energy loss of the scattered $x$-ray is characteristic of the final state, but the intensity of the emitted radiation also depends on the transition amplitude to the core-excited state. The intermediate density of states is in no way changed of course, but contributions from different intermediate states can be separated if interference between different intermediate states is neglected. This assumption is not generally satisfied, but if the manifestations of the interference can be well-understood the technique may prove uniquely powerful. 
Resonant X-ray Raman scattering was first observed by Sparks (18) using a $\mathrm{Cu}$ $\mathrm{K} \alpha$ source to irradiate a variety of targets, and subsequently investigated using synchrotron radiation (19-22). The field benefited from early theoretical developments by Tulkki and Aberg $(14,23-27)$ who described the process in terms of the resonant scattering theory and also derived a simplified model which continues to provide valuable guidance to experimentalists. The Auger analog to this process was first observed by Brown et. al. $(28,29)$, work which has been significantly expanded upon recently (30-34).

The aspects of resonant Raman scattering that make it potentially unique for spectroscopy are the narrow spectral widths of the emitted radiation (photons or electrons) and the dispersion of the emitted energy with varying incident photon energy. The former characteristic effectively enhances the resolution while the latter allows discrete states to be distinguished from continuum states. These characteristics are simple consequences of energy conservation, and discussed elsewhere $(35,36)$. Here we simply note that the important point is whether one or two bodies share energy with the atom (ion) in the final state, and in this context the reasoning is the same whether the bodies are electrons or photons. The features of radiative and nonradiative Raman scattering are compared in Table 1.

These characteristics are best illustrated with a spectrum, for which we use an Auger Raman spectrum of the $\mathrm{K}$-shell absorption edge in argon. To avoid the complicated forest of lines usually present in Auger spectra we select a single line that dominates the KLL spectrum, the $\mathrm{L}_{2} \mathrm{~L}_{3}{ }^{1} \mathrm{D}_{2}$ transition (37), and follow it's evolution as the photon energy is scanned across the $\mathrm{K}$ threshold.

Table 1. Characteristics of X-ray and Auger Resonant Raman Scattering

\section{Resonant Excitation}

X-Ray Raman

Auger Raman

- Energy of emitted $x$-ray disperses nearly $1: 1$ with incident photon energy.

- Spectral width of emitted $x$-ray includes final state lifetime and incident photon bandpass.

- Resonant peak maximum near - Resonant peak maximum greater than characteristic fluorescence energy. diagram energy due to higher charge in final state and $\mathrm{PCl}$.

\section{Ionization}

X-Ray Raman

\section{Auger Raman}

- Well above threshold, energy of characteristic fluorescence is nondispersive and the spectral width includes the natural widths of the intermediate and final states, but not the incident photon bandpass.

- Energy of emitted electron disperses nearly $1: 1$ with incident photon energy.

- Spectral width of emitted electron includes final state lifetime and incident photon bandpass.

Near threshold, the fluorescence peak is truncated on the high-energy side due to conservation of energy.

- Centroid of fluorescence peak moves due to varying cut off from E. cons.

- Observable well below threshold.

- Well above threshold, energy of the diagram line is constant and the spectral width includes the natural widths of the intermediate and final states, but not the incident photon bandpass.

- Near threshold, the diagram peak is broadened and asymmetric due to $\mathrm{PCl}$, although $E$. cons. truncation effects should be present too.

- Peak maximum shifted due to $\mathrm{PCl}$, varying cut-off expected also.

- May not contribute strongly below threshold due to $\mathrm{PCl}$ recapture. 


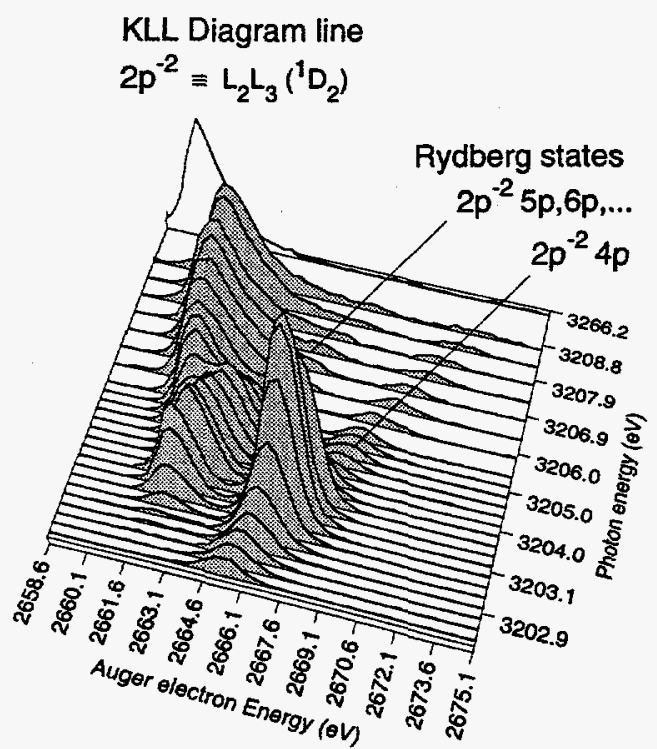

Asymmetry of $2 p^{-2} 4 p$ peak profile

Figure 3. Auger resonant Raman spectrum of the K edge of argon.

The data presented in figure 3 is a series of electron spectra of the $L_{2} L_{3}{ }^{1} D_{2}$ peak in the KLL auger spectrum as the photon energy is scanned across the $\mathrm{K}$ absorption threshold at $3206.26 \mathrm{eV}$ (38). The photon energy and electron energy scales have been set using the values of Asplund et. al. (37) and Breinig et. al. (38) respectively. The individual Auger spectra have been background subtracted and normalized to the total LMM electron yield. No smoothing is applied.

The ${ }^{1} \mathrm{D}_{2}$ diagram line measured $60 \mathrm{eV}$ above threshold is shown at the rear of the left-hand spectrum. In comparison, the line observed just above threshold is broadened, asymmetric and shifted in energy due to post-collision interaction effects (PCI). Below threshold, we observe two peaks (indicated by straight lines) resulting from resonant excitation to Rydberg states instead of ionization. The $4 \mathrm{p}$ is well resolved and shows intensity well beyond threshold, while the higher Rydbergs are not well-resolved from each other and blend into the onset of the continuum line. The $4 p$ peak is much more separated from the higher Rydbergs than in the absorption spectrum due to the resonant Auger energy shift which results from the change in nuclear charge between the intermediate and final states(39). This increases the effective resolution of resonant Auger Raman scattering relative to resonant $\mathrm{x}$-ray Raman scattering.

A simple spectator model would assume that the initially excited electron stays in the same Rydberg orbital. If so, the resonant peaks in these spectra (which show how the excitation to the final states changes as a function of the incident photon energy) would also tell us how the excitation to the intermediate states varies - thus giving the intermediate density of states with high resolution. However, while the spectator model is very useful for interpretation, it is not suitable for quantitative analysis as can be seen in the right-hand spectrum in Fig. 3. Assuming spectator relaxation we would expect the profile of the $4 \mathrm{p}$ peak as a function of photon energy to be a symmetric Voigt shape. However the peak is strongly asymmetric due to contributions from intermediate states with the excited 
electron in a $5 \mathrm{p}$ or higher orbital. This effect has been calculated by Armen et. al. (40) and their results agree well with experiment, demonstrating the need to take interference effects into account when trying to extract the intermediate density of states from such experimental results.

Understanding the effects of interference is essential to applying this technique for high resolution spectroscopy, and several groups are already studying interference effects in resonant x-ray Raman spectra (40-44). In addition, early studies of the polarization and angular distribution of resonantly scattered $\mathrm{x}$-rays proved very successful in probing the symmetries of molecular orbitals $(45,46)$, and point to many interesting future developments.

\section{Summary}

We have discussed some of the issues related to exploiting third-generation synchrotron sources to extend studies of inner-shell structure to the deepest shells of atoms. The tremendous progress in the VUV and with traditional x-ray sources has laid the ground work for such experiments, but the shift in range of photon energies also entails a shift to a broader perspective to include scattering processes as subjects of investigation. And while this range of energies presents opportunities to study new physics, it also presents new obstacles. We have endeavored to illustrate some of these challenges, as well as possible approaches to dealing with them. The author warmly acknowledges Steve Southworth and Mike MacDonald for collaboration on this and other projects. This work was supported by the U.S. Department of Energy, Office of Basic Energy Sciences, under Contract No. W-31-109-Eng-38.

\section{References}

1. Grant, I. P., in Atomic and Molecular Physics Handbook, edited by Drake, G. W. F., Woodbury, NY: AIP Press, 1996, ch. 22, pp. 258-286.

2. Hansen, P. G., Jonson, B., Borchert, G. L., and Schult, O. W. B., in Atomic Inner-Shell Physics, edited by Crasemann, B., New York: Plenum, 1983.

3. Suortti, P., in these proceedings.

4. Crasemann, B., in Atomic and Molecular Physics Handbook, edited by Drake, G. W. F., Woodbury, NY: AIP Press, 1996, ch. 60, pp. 701-711.

5. Pratt, R. H., Kissel, L., and Bergstrom, P. M., in Resonant Anomalous X-Ray Scattering, edited by G. Materlik, C. J. Sparks, K. Fischer, Amsterdam: Elsevier Science, 1994.

6. Hemmers, O., Fisher, G., Glans, P., Hansen, D.L., Wang, H., Whitfield, S.B., Lindle, D.W., Wehlitz, R., Levin, J.C., Sellin, I.A., Perera, R.C.C., Dias, E.W.B., Chakraborty, H.S., Deshmukh, P.C., and Manson, S.T., private communication.

7. Krässig, B., Jung, M., Gemmell, D. S., Kanter, E. P., LeBrun, T., Southworth, S. H., and Young, L., Phys. Rev. Lett. 5, 4736 (1996).

8. Krässig, B., in these proceedings.

9. Kissel, L., Zhou, B., Roy, S. C., Gupta, S. K. S., and Pratt, R. H., Acta Cryst. A51, 271-288 (1995).

10. Evans, R. D., in Handbuch der Physik, edited by Flügge, S., Berlin: Springer Verlag, 1958, vol. 34, pp. 218-298.

11. Bergstrom, P. M., Suric, T., Pisk, K., and Pratt, R. H., Phys. Rev. A 48, 1134 (1993).

12. Sakurai, J. J., Advanced Quantum Mechanics, New York: Addison-Wesley, 1967, ch. 2-6, pp. 56-57. 
13. Heitler, W., The Quantum Theory of Radiation, London: Oxford University Press, 1954.

14. Ảberg, T., "in Proceedings of the conference on Raman Emission by X-rays Workshop, edited by Ederer, D. L. and McGuire, J. H., 1996.

15. Chantler, C. T., J. Phys. Chem. Ref. Data 24, 71 (1995).

16. Hubbell, J. H., J. Phys. Chem. Ref. Data 23, 339 (1994).

17. Krause, M. O. and Oliver, J. H., J. Phys. Chem. Ref. Data 8, 329-337 (1979).

18. Sparks, C. J., Phys. Rev. Lett. 33, 262 (1974).

19. Eisenberger, P., Platzman, P. M., and Winick, H., Phys. Rev. Lett. 36, 623 (1976).

20. Briand, J. P., Girard, D., Kostroun, V. O., Chevalier, P., Wohrer, K., and Mossé, J. P., Phys. Rev. Lett. 46, 1625 (1981).

21. Hämäläinen, K., Siddons, D. P., Hastings, J. B., and Berman, L. E., Phys. Rev. Lett. 67, 2850 (1991).

22. MacDonald, M. A., Southworth, S. H., Levin, J. C., Henins, A., Deslattes, R. D., T.LeBrun, Azuma, Y., Cowan, P. L., and Karlin, B. A., Phys. Rev A 51, 3598 (1995).

23. Tulkki, J. and Åberg, T., J. Phys. B 15, L435-L440 (1982).

24. Tulkki, J., Phys. Rev. A 27, 3375 (1983).

25. Åberg, T. and Tulkki, J., in Atomic Inner-Shell Physics, edited by Crasemann, B., Plenum, 1985.

26. Ảberg, T. and Crasemann, B., in X-Ray Anomalous (Resonance) Scattering: Theory and Experiment, edited by Fischer, K., Materlik, G., and Sparks, C. J., Amsterdam: Elsevier/North-Holland, 1994.

27. Tulkki, J., thesis, Helsinki University of Technology, 1985 (unpublished).

28. Brown, G. S., Chen, M. H., Craseman, B., and Ice, G., Phys. Rev. Lett. 45, 1937-1940 (1980).

29. Armen, G. B., Ảberg, T., Levin, J. C., Crasemann, B., Chen, M. H., Ice, G. E., and Brown, G. S., Phys. Rev. Lett. 54, 1142 (1985).

30. Kivimäki, A., Brito, A. N. d., Aksela, S., Aksela, H., Sairanen, O. P., Ausmees, A., Osborne, S. J., Dantas, L. B., and Svensson, S., Phys. Rev. Lett. 71, 4307 (1993).

31. Wang, H., Woicik, J. C., Åberg, T., Chen, M. H., Herrera-Gomez, A., Kendelewicz, T., Mäntykenttä, A., Miyano, K. E., Southworth, S., and Crasemann, B., Phys. Rev. A 50, 1359 (1994).

32. Aksela, S., Kukk, E., Aksela, H., and Svensson, S., Phys. Rev. Lett. 74, 2917 (1995).

33. Armen, G. B. and Wang, H., Phys. Rev. A 51, 1241 (1995).

34. Drube, W., Treusch, R., and Materlik, Phys. Rev. Lett. 71, 42 (1995).

35. Cowan, P. L., in X-Ray Anomalous (Resonance) Scattering: Theory and Experiment, edited by Materlik, G., Sparks, C. J., and Fischer, K., Amsterdam: Elsevier/North-Holland, 1994.

36. LeBrun, $T$., "Interpreting $\mathrm{x}$-ray and Auger resonant Raman spectra," in Proceedings of the conference on Raman Emission by X-rays Workshop, edited by Ederer, D. L. and McGuire, J. H., 1996.

37. Asplund, L., Kelfve, P., Blomster, B., Siegbahn, H., and Siegbahn, K., Physica Scripta 16, (1977).

38. Breinig, M., Chen, M. H., Ice, G. E., Parente, F., and Crasemann, B., Phys. Rev. A 22, 520 (1980).

39. Eberhardt, W., Kalkoffen, G., and Kunz, C., Phys. Rev. Lett. 41, 156 (1978).

40. Armen, G. B., Levin, J. C., and Sellin, I. A., Phys. Rev. A 53, 772 (1996).

41. Neeb, M., Rubensson, J.-E., Biermann, M., Eberhardt, W., Randall, K. J., Feldhaus, J., Kilcoyne, A. L. D., Bradshaw, A. M., Xu, Z., Johnson, P. D., and Ma, Y., Chem. Phys. Lett. 212, 205 (1993).

42. Carra, P., Fabrizio, M., and Thole, B. T., Phys. Rev. Lett. 74, 3700 (1995).

43. Luo, Y., Agren, H., Guo, J. H., Skytt, P., Wassdahl, N., and Nordgren, J., Phys. Rev. A 52 , 3730-3736 (1995).

44. Gel'mukhanov, F. and Agren, H., J. Phys. B 29, 2751-2762 (1996).

45. Lindle, D. W., Cowan, P. L., Jach, T., LaVilla, R. E., and Deslattes, R. D., Phys. Rev. A 43, 2353-2366 (1991).

46. Southworth, S. H., Lindle, D. W., Mayer, R., and Cowan, P. L., Phys. Rev. Lett. 67, 10981101 (1991). 